\title{
A boundary element method for a second order elliptic partial differential equation with variable coefficients
}

\author{
W. T. Ang \\ Faculty of Engineering, Universiti Malaysia Sarawak, Malaysia \\ J. Kusuma \\ Department of Mathematics, Universitas Hasanuddin, Indonesia
}

\&

D. L. Clements

Department of Applied Mathematics, The University of Adelaide, Australia

(Received 15 December 1995; revised version received 15 August 1996; accepted 3 October 1996)

\begin{abstract}
A boundary element method is derived for solving a class of boundary value problems governed by an elliptic second order linear partial differential equation with variable coefficients. Numerical results are given for a specific test problem. (C) 1997 Elsevier Science Ltd
\end{abstract}

Key words: Boundary element method, heterogeneous media, inhomogeneous elasticity.

\section{INTRODUCTION}

The second order elliptic partial differential equation

$$
\frac{\partial}{\partial x}\left[K(x, y) \frac{\partial \phi}{\partial x}\right]+\frac{\partial}{\partial y}\left[K(x, y) \frac{\partial \phi}{\partial y}\right]=0, \quad \phi=\phi(x, y)
$$

with $K(x, y)$ being a suitably given function of the independent variables $x$ and $y$, occurs in certain classes of thermostatic, elastostatic, electrostatic and seepage problems where the media involved exhibit heterogeneous properties. Of particular interest is the boundary value problem which requires solving eqn (1) in a finite region $R$ in the $0 x y$ plane subject to the conditions

$$
\begin{array}{rlrlr}
\phi & =u(x, y) & \text { on } & & \mathcal{C}_{1} \\
\frac{\partial \phi}{\partial \mathbf{n}} & =v(x, y) & \text { on } & & \mathcal{C}_{2}
\end{array}
$$

where $C=\mathcal{C}_{1} \cup \mathcal{C}_{2}$ is the boundary of the region $R, u$ and $v$ are suitably prescribed functions of $x$ and $y$, and $n$ denotes the unit outward normal vector to $C$.
For the special case where $K(x, y)$ is a constant function throughout $R$, (1) reduces to Laplace's equation and the boundary element method for solving this boundary value problem is well established (see for example, Brebbia and Walker ${ }^{1}$ ).

However, due to the inherent mathematical difficulties in obtaining the fundamental solution, the boundary element method has not been widely used to solve this boundary value problem for the general case where $K=K(x, y)$ varies continuously throughout $R$. The papers which have addressed this problem have considered special cases of (1). Clements ${ }^{2}$ used a series solution to obtain a boundary integral solution to (1) for the case when $K$ depends only on one of the two independent variables. Cheng ${ }^{3,4}$ considered the special case when $K$ $(x, y)$ takes a form which allows (1) to be transformed to Laplace's equation or the Helmholtz equation. Rangogni ${ }^{5}$ used a perturbation technique to obtain a boundary integral solution to (1) for the case when $K$ exhibits small variations from a constant value. More recently Shaw, ${ }^{6}$ Shaw and Gipson ${ }^{7,8}$ and Gipson et al. ${ }^{9}$ have also considered the boundary element method in 